\title{
Status of neurosurgery for mental disorder
}

\section{in Scotland}

\author{
Selective literature review and overview of current clinical \\ activity
}

KEITH MATTHEWS and MUFTAH S. ELJAMEL

\begin{abstract}
Background Despite the application of ablative neurosurgical treatments for intractable mental disorder throughout most of the past century, unequivocal evidence for efficacy has not been provided.
\end{abstract} neurosurgery for mental disorder and to describe the activities of the Scottish national service.

Method Relevant literature is reviewed alongside a description of recent clinical activity.

Results Neurosurgical treatment is offered to a small number of patients severely disabled by otherwise intractable mental disorder. There are inequalities in the strength of evidence to support the use of some of these procedures. The frequency and severity of adverse effects remains unclear.We are collecting data that should inform future practice.

Conclusions Modern neurosurgery can offer clinically meaningful symptom relief and improved function for 'untreatable' patients with chronic, severe depression and obsessive-compulsive disorder. However, follow-up studies of greater rigour are required. The potential role of non-ablative alternatives remains unclear.

Declaration of interest K.M. has received payment for lectures on the management of depression from various pharmaceutical companies.
In 1993 a Good Practice Group was established by the Scottish Executive (then Office) Clinical Resource and Audit Group (CRAG) Working Group on Mental Illness to evaluate the need for a Scottish neurosurgical service for the treatment of chronic, intractable mental disorder (CRAG Working Group on Mental Illness, 1996). Having reviewed a range of complex ethical, legal and clinical considerations, the key recommendations were:

(a) neurosurgery for mental disorder should continue to be available in Scotland, but only as a treatment for intractable obsessive-compulsive disorder and affective disorders (for example major depressive illness);

(b) there ought to be a national Standing Advisory Committee;

(c) an annual report should be made to the Committee by the service providers.

A framework for best practice was agreed and published in 1996; the second recommendation was enacted towards the end of 2001, and the first report has been published on the Scottish Executive Health Department's website (Matthews \& Eljamel, 2001). We here describe the status of neurosurgery for mental disorder in Scotland.

\section{ROLE OF NEUROSURGERY IN MODERN PRACTICE}

Originally introduced as a treatment for schizophrenia in the era before the discovery of effective drug treatments (Moniz, 1936), the frontal or prefrontal lobotomy came to be widely used as a treatment for a broad range of poorly specified mental diseases and behavioural disturbances. These crude and destructive freehand procedures were overused, with an absence of critical appraisal of their efficacy and adverse effects. Nevertheless, with refinement of the precision and targeting of surgery - notably the application of stereotactic neurosurgical techniques - evidence has accrued to support the use of irreversible focal tissue ablation in the management of a few patients with severely disabling defined psychopathological conditions. Although different centres have employed different neurosurgical techniques to target different structures, all modern neurosurgery for mental disorder involves the ablation, or disconnection, of ventral and medial prefrontal cortical areas. This is achieved by the precise placement of small lesions in target structures that are considered to be relevant to the disorder. These targets evolved on an empirical basis, but recent structural, functional and neuroanatomical advances confirm that both the main clinical indications for neurosurgery-chronic intractable depression and obsessive-compulsive disorder (OCD) - are associated with neurobiological changes within these regions or in key projection areas (Sheline et al, 1996; Drevets et al, 1997; Ongur et al, 1998; Rajkowska et al, 1999; Szeszko et al, 1999). Existing non-surgical treatments remain demonstrably inadequate for significant numbers of people affected by these conditions. Up to $40 \%$ of patients with depression fail to respond to first-line antidepressant drug treatments, and of those who do respond, only a modest proportion achieve full recovery (Judd, 1997). Between $5 \%$ and $15 \%$ of depressive episodes become chronic (>24 months) and potentially $1.5 \%$ of the general population suffer from chronic depression (Judd, 1997). Furthermore, treatment responsiveness decreases with increasing duration of illness and number of previous episodes of depression. Hence, there are many for whom neither drug treatments nor psychological therapies restore function. Similarly, although the clinical course of OCD is highly variable (Skoog \& Skoog, 1999), and most patients experience meaningful symptomatic relief, at least in the short term, with exposure to modern antidepressant drug treatments and/or focused psychological interventions, the longitudinal clinical course appears disappointingly stable (MataixCols et al, 2002). Indeed, for men with early onset of illness and poor social adjustment, research suggests that unremitting symptoms are likely (Skoog \& Skoog, 1999).

\section{DOES NEUROSURGERY WORK?}

These are numerous reviews of the outcome of neurosurgery for mental disorder. 
The consistency of the conclusions offered is remarkable - particularly given the heterogeneity of the populations studied, the neurosurgical procedures used and the outcome measures employed. A detailed critical review of the literature can be found within the Royal College of Psychiatrists' report on neurosurgery for mental disorder (Royal College of Psychiatrists, 2000). Therefore, only a brief review of salient aspects is presented here.

The accumulated literature on neurosurgery for mental disorder remains highly unsatisfactory. There has been no prospective, randomised, double-blind, placebocontrolled trial of any procedure, and none is likely. To perform sham intracranial surgery, with all its associated hazards, would probably be unethical. It is also unlikely that a representative sample of prospective neurosurgery candidates would consent to randomisation, when they invariably view the procedure as their 'last resort'. Although it is generally believed that placebo response rates are low with chronic intractable illnesses, there can be few interventions with greater potential for eliciting such responses. Indeed, early responses that are not sustained $-\mathbf{a}$ potential placebo response - are not uncommon (see, for example, Poynton et al, 1995). The observed and reported clinical responses to neurosurgery are almost certainly the combined product of surgery-specific and non-specific influences. However, that placebo or other non-specific effects might account for consistent reports of long-term clinical improvement seems implausible. If there is continuing, unjustified exaggeration of the efficacy of neurosurgery, this is more likely to be attributable to reporting bias than to placebo response (Hrobjartsson \& Gotzsche, 2001).

A common weakness of published outcome studies is the absence of demonstrably independent assessments. Generally, outcome has been assessed by service providers (with obvious potential for bias) and has not been masked. Also, many studies can be criticised for the short duration of the follow-up period and the failure to acquire data from all patients. Outcome measures have usually been categorical global functioning scales, providing limited detail on crucial domains such as residual symptom burden, social and occupational functioning and perceived quality of life. Studies have rarely commented upon the quality of the neurosurgical interventions; for example, the size and placement of lesions are rarely described. There is no report of sustained benefit following either deliberate sham or accidentally misplaced lesions. The absence of anatomical confirmation is a major deficiency of many older studies in which the neurosurgery was not conducted under stereotactic guidance. Indeed, it may also be of major relevance for stereotactic neurosurgery (Lippitz et al, 1999). Similarly, there are usually few (if any) details provided of postoperative management. Even when rapid and extensive symptom relief follows neurosurgery, significant psychological and social adjustments are inevitably required.

\section{WHAT EVIDENCE SUPPORTS THE USE OF NEUROSURGERY?}

To address the question of efficacy without the use of prospective, randomised trials, we must evaluate alternative study designs. For example, there are studies that have employed retrospective control groups. Although there are many potential interpretative difficulties, each study suggested improved outcome following neurosurgery: leucotomy for agoraphobia (Marks et al, 1966); leucotomy for obsessional disorders (Tan et al, 1971); subcaudate tractotomy for depression and OCD (Bridges \& Goktepe, 1973); orbitomedial and cingulate lesions for OCD (Hay et al, 1993); and subcaudate tractotomy or multifocal leucocoagulation for OCD (Cosyns et al, 1994). Consolidated reviews of different neurosurgical procedures, deployed for different clinical indications, without anatomical quality assessments and based on global categorical outcome measures, suggest that over half of patients derived significant benefit from neurosurgery, with around one-third experiencing dramatic functional improvements (Kiloh et al, 1988; Waziri, 1990). Unfortunately, it is impossible to draw reliable conclusions regarding the comparative efficacy of different procedures.

For the treatment of intractable depressive disorder, although several procedures have been deployed, the overwhelming majority of data concern a procedure no longer performed - the subcaudate tractotomy by focal irradiation (Knight, 1965; Bridges et al, 1994). Although a substitute method has been described obviating the need to secure a supply of radioactive yttrium-90 rods (Malhi \& Bartlett, 1998), the Geoffrey Knight Unit in London no longer offers neurosurgery for mental disorder. The two remaining centres in the UK (Dundee and Cardiff) have favoured anterior capsulotomy (bilateral thermal lesions of the anterior limbs of the internal capsule): or anterior cingulotomy (bilateral thermal lesions of the anterior cingulate gyri) for such patients (Figs 1 and 2). However, the efficacy of the anterior capsulotomy procedure for depressive disorder is poorly established. The most informative data are those presented by Herner (1961), who reported that 9 of 19 anterior capsulotomy procedures were associated with substantial benefit when patients were followed over a period of up to 24 months; 4 patients were reported to have deteriorated. Thus, the evidence base for performing anterior capsulotomy for depression is restricted to a single, 40-year-old, open

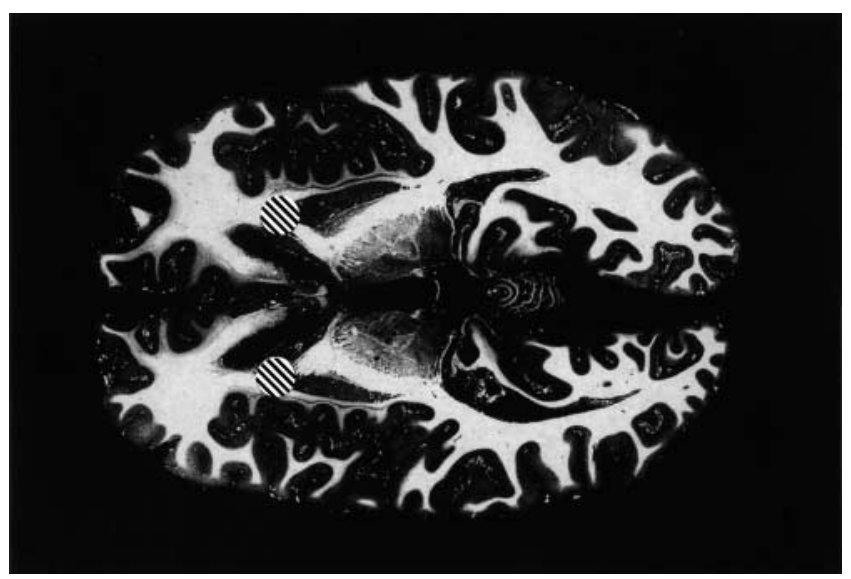

Fig. I Anterior capsulotomy. Horizontal section through human brain at the level of the basal ganglia; fibres appear white, cellular material is stained dark grey. The marked areas $\$$ represent the site and extent of typical thermal anterior capsulotomy lesions. 


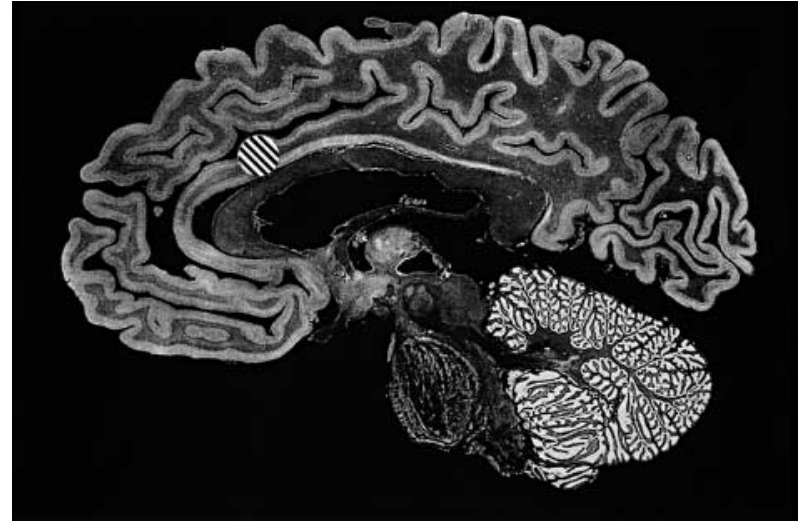

Fig. 2 Anterior cingulotomy. Sagittal section through human brain exposing the medial cerebral hemisphere

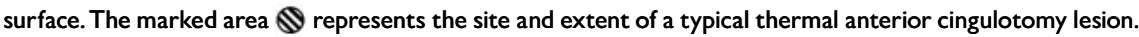

study of 19 patients of uncertain diagnostic status. With the Dundee and Cardiff neurosurgery services having performed over 50 such procedures for depressive disorder within the past decade, there is a compelling case for a collaborative outcome study. In Dundee, we offer anterior cingulotomy for resistant depressive disorder, in the light of the favourable outcomes reported by other centres (e.g. Ballantine et al, 1987; Spangler et al, 1996) and our own clinical experience. For intractable OCD, although subcaudate tractotomy and multifocal leucocoagulation have been reported to offer benefit (reviewed by the Royal College of Psychiatrists, 2000), anterior capsulotomy (Waziri, 1990) and anterior cingulotomy (Dougherty et al, 2002) are the best-established current treatment options. Although there is no compelling evidence either way, it is possible that anterior capsulotomy may offer marginal superiority.

\section{ADVERSE EFFECTS OF NEUROSURGERY}

Advances in neuroimaging techniques and stereotactic targeting technology (stereotactic frames and software) have made it possible to place millimetre-scaled lesions accurately and precisely in neural structures implicated in the symptoms of depression and OCD. The same advances have also made it possible to define the topography of lesions and to correlate this with clinical effects. The adverse effects associated with neurosurgery for mental disorder can be considered under two categories: general risks associated with any intracranial surgery, and risks specific to the particular procedure carried out.
The main general risks are those of vascular events, confusional states and postoperative epilepsy. The surgical death rate in neurosurgery for mental disorder is extremely low and no higher than for stereotactic intracranial surgery performed for other indications. The current risk of acquiring major neurological deficit (usually by vascular injury) as a consequence of frame-based stereotactic surgery is less than $1 \%$. Transient confusional states ( $<21$ days) are not uncommon following intracranial neurosurgery, with increased rates following larger, nonstereotactic lesions and in elderly patients. Rates following anterior capsulotomy and anterior cingulotomy are very low. Epilepsy rates are also low following stereotactic neurosurgery, even where lesions are large. We inform potential patients that the risk of at least one seizure following anterior cingulotomy (with no previous history of epilepsy) approaches $10 \%$ over 10 years (Jenike et al, 1991). However, such seizures are generally responsive to anticonvulsant monotherapy. Weight gain of clinical significance has been reported with both anterior capsulotomy (Herner, 1961) and subcaudate tractotomy procedures (Bridges et al, 1994), but not with anterior cingulotomy. It is, of course, difficult to dissociate the impact of neurosurgery from that of other influences such as changes in general levels of activity, medication and catering arrangements.

Despite concerns that surgical interference with the frontal lobes must lead to adverse personality change, there is surprisingly little evidence to support its occurrence with modern neurosurgery. Indeed, there is some evidence to the contrary (e.g. Mindus \& Nyman, 1991). Personality changes following lobotomy, or other widespread lesions, would have been very common. Reports of adverse change following neurosurgery for mental disorder (for example, the development of irritability, aggression and loss of responsiveness to social cues) are allegedly infrequent (0.4$4 \%$; Kiloh et al, 1988), even in patients treated with large frontal lesions such as subcaudate tractotomy $(<7 \%$; Goktepe $e t$ al, 1975). There is one report of adverse personality change following anterior cingulotomy, where 3 of $27(11 \%)$ patients were considered to show postoperative 'indifference' or 'lack of judgement' (Vilkki, 1977). It must be noted, however, that valid, sensitive and repeatable methods for the measurement of personality are lacking, and underestimation is likely.

There is no evidence that stereotactic neurosurgery for mental disorder results in significant intellectual impairment. Indeed, improvements in general measurements of IQ have been reported (Kelly, 1980), presumably following improved attentional capacity associated with symptom relief. Although some measures of frontal functioning have detected transient impairments with some neurosurgical procedures (Kartsounis et al, 1991), evidence for enduring deficit is scant. However, sensitive, specific tests of frontal function have not generally been applied. Three more-recent reports have highlighted previously unrecognised adverse effects. Two single-case studies suggest that anterior capsulotomy may exert subtle adverse effects on social insight (Happe et al, 2001) and that anterior cingulotomy may impair attentional and executive processes (Ochsner et al, 2001). In a 20-year follow-up of 16 patients who received stereotactic ventromedial frontal leucotomy for intractable OCD, 8 developed significant substance dependence postoperatively (Irle et al, 1998). Clearly, each of these observations requires detailed evaluation in other neurosurgical series.

\section{DOES NEUROSURGERY DESTROY 'HEALTHY' BRAIN TISSUE?}

Ablative neurosurgery has always been subject to special consideration by the medical and legal communities, as well as by the public, because of the perception that it is controversial, hazardous and irreversible. The essence of the controversy has always been that neurosurgery for mental disorder 
represents a destructive 'physical' treatment for 'psychological' disorders in which the brain tissue targeted for ablation is 'healthy'. Indeed, the recently published Millan Committee report on proposed reforms to the Mental Health (Scotland) Act 1984 retains this outdated, dualist perspective: 'we agree that any operation to destroy brain tissue, where this is not for treatment of a physical illness, requires stringent safeguards' (Millan Committee, 2001). The importance of recent research demonstrating discrete changes in cerebral metabolism and anatomical structure in defined mental disorders (Sheline et al, 1996; Drevets et al, 1997; Ongur et al, 1998; Rajkowska et al, 1999; Szeszko et al, 1999) cannot be overstated. Destructive and irreversible neurosurgical treatments are used without legal provision or restriction to manage distressing and treatment-refractory medical problems such as the motor symptoms of Parkinson's disease, epilepsy and chronic pain. The techniques and technologies used to perform such surgery are identical to those used in neurosurgery for mental disorder. Conceptually, they are also identical - specific areas of the brain are destroyed, or functionally inactivated, to alleviate otherwise intractable symptoms. With rapidly accruing evidence from multiple sources that depression and OCD are associated with distinctive, regionally specific changes in brain function and structure, we must consider at which point these conditions cease to be 'non-physical'. To assert that the brain of an individual with chronic, severe depression or OCD represents 'healthy' tissue now seems illjudged and implausible. We can no longer conclude that neurosurgery for mental disorder is targeting 'healthy' tissue.

\section{THE DUNDEE SERVICE}

The first modern neurosurgical procedures for mental disorder performed in Dundee took place in 1992. The clinical service was established by Professor George Fenton (Department of Psychiatry, University of Dundee) and Mr T. R. K. Varma (Department of Surgical Neurology, Dundee Royal Infirmary). Between January 1990 and December 2001, a total of 34 such procedures were performed (Table 1). Since its inception, the configuration and activities of the service have evolved to reflect the need for expert multi-disciplinary input. For example, assessment of the adequacy of previous psychological treatments and of potential suitability for further psychological therapy before consideration for neurosurgery now involves detailed, independent assessment by a senior academic clinical psychologist and a consultant psychotherapist. Operational policies for neurosurgery for mental disorder have been extended and refined following discussion and correspondence with other specialist treatment services for intractable depression and OCD within the UK. Independent clinical assessments are provided for all patients by representatives from the Mental Welfare Commission for Scotland. Such assessments include a consideration of diagnostic issues, the degree of functional impairment, the adequacy of previous treatments, the suitability of the proposed rehabilitation plan and the appropriateness of neurosurgery.
These assessments are provided as a statutory requirement for all patients liable to detention under UK mental health legislation and as an informal agreement for all non-detained patients. Mental Welfare Commission assessments are conducted by a group of three commissioners with at least one medical representative. Thus, before proceeding to neurosurgery, the individual's suitability and capacity to provide informed consent will have been assessed by the referring mental health team (including the consultant psychiatrist and the responsibile medical officer), the Dundee team and the Mental Welfare Commission for Scotland.

On receipt of a formal, written referral, the assessment procedure begins. This may vary for geographical reasons and according to the nature of the unit making the referral. Psychiatric assessment can be conducted at home, at the patient's base hospital, or during a period of inpatient assessment in Dundee. Communitybased assessment facilitates scrutiny of local medical and psychiatric case records, collection of information from relatives and from members of the local health care team. In-patient assessment is preferred when the source of referral is geographically distant and it is necessary to complete a detailed review of previous psychological treatment methods and responses, particularly for patients referred from centres with limited access to such treatment methods. The aims of the initial assessment are to confirm diagnosis, to determine the appropriateness, vigour and adequacy of previous treatments, and to consider the impact of illness on

Table I Activity of the Dundee neurosurgery for mental disorder programme 1990-200I: referral and operation rates, separated by gender

\begin{tabular}{|c|c|c|c|c|c|c|c|c|c|c|c|c|c|c|c|c|c|c|c|c|c|c|c|c|c|c|}
\hline & \multicolumn{2}{|c|}{1990} & \multicolumn{2}{|c|}{ |99| } & \multicolumn{2}{|c|}{1992} & \multicolumn{2}{|c|}{1993} & \multicolumn{2}{|c|}{1994} & \multicolumn{2}{|c|}{1995} & \multicolumn{2}{|c|}{1996} & \multicolumn{2}{|c|}{1997} & \multicolumn{2}{|c|}{1998} & \multicolumn{2}{|c|}{1999} & \multicolumn{2}{|c|}{2000} & \multicolumn{2}{|c|}{2001} & \multicolumn{2}{|c|}{ Total } \\
\hline & $M$ & $F$ & $M$ & $F$ & $M$ & $F$ & $M$ & $F$ & $M$ & $F$ & $M$ & $F$ & $M$ & $F$ & $M$ & $F$ & $M$ & $F$ & $M$ & $F$ & $M$ & $F$ & $M$ & $F$ & $M$ & $F$ \\
\hline $\begin{array}{l}\text { Referred for consideration } \\
\text { for NMD (n) }\end{array}$ & I & 0 & 0 & 0 & 3 & 3 & I & 2 & 0 & 5 & I & 2 & 0 & 4 & 3 & 6 & 4 & 4 & 4 & 2 & 8 & 9 & 7 & II & 32 & 48 \\
\hline \multicolumn{27}{|l|}{ Procedure (n) } \\
\hline $\begin{array}{l}\text { Bilateral anterior } \\
\text { capsulotomy }\end{array}$ & - & - & - & - & I & $\mathrm{I}$ & 1 & 4 & - & I & - & 3 & I & I & - & 3 & 3 & 3 & I & 2 & - & - & - & - & 7 & 18 \\
\hline $\begin{array}{l}\text { Bilateral anterior } \\
\text { cingulotomy }\end{array}$ & - & - & - & - & - & - & - & - & - & - & - & - & - & - & - & I & - & - & I & - & - & I & 1 & 3 & 2 & 5 \\
\hline $\begin{array}{l}\text { Vagus nerve stimulator } \\
\text { implant }\end{array}$ & & & & & & & & & & & & & & & & & & & & & & & - & 1 & - & I \\
\hline Total & & & & & & & & & & & & & & & & & & & & & & & & & 9 & 24 \\
\hline
\end{tabular}

F, female; M, male; NMD, neurosurgery for mental disorder. 
physical and mental status, social and occupational function, and quality of life. Almost all patients referred for neurosurgery for mental disorder are then directed towards treatment strategies that have not previously been explored or that have been of dubious adequacy. The major inclusion and exclusion criteria for consideration for neurosurgery are detailed in Appendix 1. These criteria are used to guide clinical decision-making, but are not applied rigidly. Indeed, the criteria are subjected to regular review to ensure that treatment advances are incorporated as necessary. We have adopted the view that the presence of a personality disorder (the presence of significant maladaptive personality traits prior to the development of mental disorder) might represent a relative contraindication to surgery. Our screening has identified three candidates for neurosurgery for mental disorder who met criteria for a diagnosis of personality disorder: however, none met criteria for treatment adequacy and none has had neurosurgery.

\section{WHEN IS PREVIOUS TREAT MENT ADEQUATE?}

Appendix 2 details the framework for determination of the adequacy of previous physical treatments. The guiding principle is that it is necessary to determine whether patients have been exposed to a sufficiently broad range of different treatments (pharmacological and psychological), in adequate 'dosage', for adequate periods. The reader will be able to identify drug treatment strategies, particular combination treatments, that do not appear on the Dundee framework. These treatments are not listed as 'required' because evidence for their efficacy falls below that for neurosurgery itself. However, it should be appreciated that most people referred for neurosurgery for mental disorder have been exposed to many more drug treatments and combinations than are listed. The most problematic assessments usually concern the adequacy of previous psychological treatments. In general, judgements involve detailed scrutiny of previous treatment records, an assessment of the likely responsiveness in terms of presenting characteristics and, in some cases, a brief trial of a potentially suitable treatment approach. Although there are specific psychological treatment approaches with proven efficacy in depressive disorder and OCD (although not for presentations of such complexity and chronicity), the availability of suitably trained, expert therapists varies from one region to another. We place particular emphasis upon adequate trials of expertly delivered cognitive-behavioural therapy. Although there is evidence for the efficacy of interpersonal therapy in less severe depressive disorder, suitably trained therapists are even more difficult to access than cognitive-behavioural therapists. Hence, interpersonal therapy does not feature as a mandatory requirement. Although there is no compelling evidence to support the efficacy of psychodynamically oriented psychological treatment approaches, an assessment of previous treatment exposures and potential suitability for subsequent treatment by such methods is routinely conducted. To date, none of those screened has been considered a suitable candidate for such treatment. Indeed, the patients referred for consideration for neurosurgery are almost invariably too ill to engage in therapy, or have previous experience of therapy leading to worsening of symptoms and intense personal distress.

\section{ALTERNATIVES TO ABLATIVE NEUROSURGERY}

Two potentially 'non-destructive' alternatives to ablative neurosurgery have emerged: vagus nerve stimulation and deep brain stimulation. The former, an approved and effective treatment for refractory epilepsy, involves intermittent electrical stimulation of afferent fibres within the vagus nerve. This has been reported to achieve a significant improvement in symptom burden and function after 12 weeks of stimulation in 18 people who were participants in an open study of 59 patients with chronic, refractory depression (Rush et al, 2000). Of this cohort, 13 of 28 were reported to have met criteria for response after 12 months of stimulation. However, there are early indications that the most chronic, treatment-refractory study entrants (i.e. those most similar to those referred for neurosurgery for mental disorder in the UK) exhibited the poorest responses to vagus nerve stimulation (Sackeim et al, 2001). Similarly, there is a single open study reporting short-term efficacy for the application of deep brain stimulation in OCD (Nuttin et al, 1999). With electrical stimulation electrodes sited bilaterally within the anterior limbs of the internal capsule, three of four patients with refractory OCD experienced benefit from deep brain stimulation over a period of weeks. Although the same technology has successfully replaced some ablative neurosurgical procedures for the management of pain and some of the symptoms of refractory Parkinson's disease, there are no longerterm follow-up data to support its use in mental disorder. Nevertheless, such procedures may offer promise for the development of reversible surgical treatments for refractory mental disorder.

\section{CAPACITY FOR INFORMED CONSENT}

Perhaps the most controversial of the recommendations made by the Good Practice Group was that neurosurgery for mental disorder 'should be able to be performed on patients who are mentally incapable of giving consent to it, provided they do not resist or oppose it' (CRAG Working Group on Mental Illness, 1996). With the recent implementation in Scotland of Part 5 of the Adults with Incapacity (Scotland) Act 2000 , much media interest has focused on this proposal. As the sole service in Scotland providing neurosurgery for mental disorder, we wish to clarify our position while awaiting parliamentary affirmation of law. Treatment of some forms of mental disorder in the absence of consent is an established principle in mental health care. However, a critical aspect of such treatments is their 'reversibility' and limited potential for serious adverse consequences. Essentially, such treatments are considered not to result in permanent changes in brain structure or function. Clearly, this does not describe neurosurgery. A body of evidence suggests that neurosurgery does improve outcome for some sufferers; however, we have no ability as yet to predict which patients might benefit. We also know that neurosurgery for mental disorder is not without risk: serious adverse events can and do occur. We cannot reliably predict these. Therefore, while aware of the moral, ethical and other arguments, the Dundee service has decided to maintain a policy of not offering neurosurgery for mental disorder to anyone who is incapable of providing sustained, informed consent. 


\section{CONCLUSIONS}

We must continue to evaluate the efficacy of neurosurgery for mental disorder against a constantly evolving knowledge base, both in terms of the underlying neuroscience of mental disorder and with respect to advances in treatment. It is also necessary rigorously to evaluate 'reversible' alternatives such as vagus nerve and deep brain stimulation. It is our view (and our experience) that neurosurgery still represents a potentially useful strategy to augment the management of chronic depression and OCD in a small number of patients. Like all other treatments for these two conditions, neurosurgery is not curative. There is a compelling need to review the long-term outcomes of the Dundee and Cardiff cohorts who have undergone neurosurgery for mental disorder.

\section{APPENDIX}

\section{Criteria for consideration for ablative neurosurgery}

Treatment-refractory depression

\section{Inclusion criteria}

(a) Age $>20$ years.

(b) Legal status: both formal and informal patients can be considered.

(c) Confirmation of diagnosis: the individual will fulfil ICD-10 criteria for one of the following:

F32.2 severe depressive episode without psychotic symptoms

F32.3 severe depressive episode with psychotic symptoms

F33.I-F33.3 recurrent depressive disorder, current episode moderate to severe

F31.4-F31.5 bipolar affective disorder, current episode severe depression with or without psychotic symptoms.

(d) Duration of illness: an absolute minimum of 3 years, with at least 2 years of unremitting symptoms despite treatment. Only in exceptional circumstances would a duration of illness of $<5$ years be considered.

(e) Consent: the patient must be capable of providing sustained, informed consent.

\section{Exclusion criteria}

(a) Age $<20$ years.

(b) Failure to fulfil ICD-10 criteria for F32.2, F32.3, F33.I-F33.3,F3I.4-F3I.5

(c) Incapacity to give sustained, informed consent.

(d) A current diagnosis of substance misuse fulfilling criteria for ICD-10 FIO-FI9 'Mental and behavioural disorders due to psychoactive substance use'.

(e) A diagnosis of organic brain syndrome fulfilling criteria for ICD-10 F00-F09, including Alzheimer's disease, vascular and other dementias. (f) A diagnosis of disorder of adult personality fulfilling criteria for ICD-10 F60-F69.

(g) A diagnosis of pervasive developmental disorder fulfilling criteria for ICD-10 F84.

\section{Treatment-refractory obsessive-compulsive} disorder

\section{Inclusion criteria}

(a) Age $>20$ years.

(b) Legal status: both formal and informal patients can be considered.

(c) Confirmation of diagnosis: individuals will normally fulfil criteria for a primary diagnosis according to ICD-10 F42.0-F42.9. Individuals with treatment-refractory obsessional and/or compulsive symptoms in the presence of other comorbid mental disorder (e.g. depression, schizophrenia) can be considered for surgery but additional criteria for adequacy of treatment will be applied.

(d) Duration of illness: an absolute minimum of 3 years, with at least 2 years of unremitting symptoms despite intensive psychopharmacological and psychological treatment. Only in exceptional circumstances would a duration of illness of $<5$ years be considered.

(e) Consent: the patient must be considered capable of providing sustained, informed consent.

\section{Exclusion criteria}

(a) Age $<20$ years.

(b) Failure to fulfil ICD-10 criteria for F42.0-F42.9.

(c) Incapacity to give informed consent.

(d) A current diagnosis of substance misuse fulfilling criteria for ICD-I0 FIO-FI9, 'Mental and behavioural disorders due to psychoactive substance use'.

(e) A diagnosis of organic brain syndrome fulfilling criteria for ICD-I0 F00-F09, including Alzheimer's disease, vascular and other dementias.

(f) A diagnosis of disorder of adult personality fulfilling criteria for ICD-10 F60-F69.

(g) A diagnosis of pervasive developmental disorder fulfilling criteria for ICD-10 F84.

(h) Absence of an adequate therapeutic trial of psychological treatment methods.

(i) Absence of adequate therapeutic trials of psychopharmacological treatment methods.

\section{APPENDIX 2}

\section{Assessment of treatment adequacy}

\section{Treatment-refractory depression}

As a guiding principle, all physical treatments that have been shown to be effective in randomised, controlled trials must have been tried in adequate dosage for an adequate period. In general terms, this will reflect the prescription of antidepressant drugs within, or above, the dose range recommended by the British National Formulary (BNF) for a period of at least 6 weeks. At present, the use of plasma drug concentration monitoring (where possible) is not included as a mandatory requirement, but is clearly desirable and may become obligatory. Most patients referred for assessment will have been exposed to many different treatment trials. The following represent those deemed essential before proceeding to neurosurgery:

(a) At least two courses of treatment with a tricyclic antidepressant drug.

(b) At least one course of treatment with a selective serotonin reuptake inhibitor.

(c) At least one course of treatment with a non reversible monoamine oxidase inhibitor.

(d) At least one of the above plus lithium augmentation for a period of 4-6 weeks, with a 12 -h postmedication plasma level of $0.4-0.8 \mathrm{mmol} / \mathrm{l}$.

(e) At least one course of treatment with an antidepressant drug as defined above, plus the prescription of a typical or atypical antipsychotic drug for a period of 6 weeks at a dose within the BNF recommended range (where psychotic symptoms are prominent in the clinical presentation, trials of both typical and atypical drugs should be performed).

(f) At least two trials of electroconvulsive therapy spaced 6 months apart. Adequacy is defined as a minimum of eight bilateral applications of the therapy with recorded evidence of seizure duration exceeding $15 \mathrm{~s}$ per treatment. Failure to respond is defined as no clinical response, minimal clinical response, or a brief response with relapse within a period of 4 weeks, despite antidepressant maintenance treatment.

(g) At least one of the following for a period of at least 6 weeks:

(i) combination therapy with clomipramine lithium and L-tryptophan; clomipramine to be administered at the maximum tolerated dose (150-250 mg per day), with a I2-h post-medication plasma lithium level of $0.4-0.8 \mathrm{mmol} / \mathrm{l}$;

(ii) combination therapy with phenelzine, lithium and L-tryptophan; phenelzine to be administered at the maximum tolerated dose (45$90 \mathrm{mg}$ per day), with a $12-\mathrm{h}$ post-medication plasma lithium level of $0.4-0.8 \mathrm{mmol} / \mathrm{l}$.

Alternative drug treatment strategies are desirable, but not essential. For each, there may be either an absence of unequivocal evidence of efficacy, or restricted suitability for selected patients on the basis of increased risk to physical health.

(a) Prescription of at least one antidepressant drug at a dosage beyond the BNF recommended maximum daily dose, with regular physiological monitoring; for example, gradual escalation to highest tolerated dose of venlafaxine $(>500 \mathrm{mg}$ per day). Alternatively, gradual escalation to highest tolerated dose of imipramine ( $>300 \mathrm{mg}$ per day). Measurement of plasma levels may be indicated, with a target concentration of 200 $250 \mathrm{ng} / \mathrm{ml}$. This level ought to be maintained for 6 weeks. 
(b) Thyroid hormone augmentation of antidepressant drug treatment. This involves the administration of liothyronine sodium $/ T_{3}$ hormone (not $T_{4}$ ) to augment the action of a tricyclic antidepressant. The tricyclic drug ought to be given at the maximum tolerated dose and then $T_{3}$ is added (increasing to $20 \mu \mathrm{g}$ three times daily).Where the patient is known to suffer from hypothyroidism and is taking replacement $T_{4}$ (biochemically euthyroid), the strategy of $T_{3}$ augmentation is still advised.

(c) Anticonvulsant drugs. Some evidence exists for the efficacy of carbamazepine and lamotrigine in resistant bipolar depression. Carbamazepine or lamotrigine ought to be prescribed either in combination with a tricyclic drug, or on their own at a dose of 800-1200 mg (carbamazepine) or $200 \mathrm{mg}$ (lamotrigine) daily in divided dose. Plasma level monitoring may be helpful with carbamazepine.

(d) Psychostimulant drug treatment. Patients ought to be exposed to the maximum tolerated dose of a tricyclic drug, to which methylphenidate is added, initially as a single $10-\mathrm{mg}$ test dose, gradually increasing to $30 \mathrm{mg}$ three times daily.

Psychological treatment methods. At least one sustained trial of structured, manualised, cognitivebehavioural therapy of 20 sessions duration (with either a cognitive or a behavioural emphasis), with long-term follow-up. Treatments ought to be delivered by a therapist with British Association for Behavioural and Cognitive Therapies (BABCP) accreditation. Where there is significant doubt over the adequacy of previous trials of psychological treatment, it may be appropriate to offer the patient at least a brief trial of a suitable psychological therapy. In some cases, this might suggest that a more intensive course of therapy ought to be instigated either in Dundee or elsewhere.

\section{Treatment-refractory obsessive-compulsive disorder}

As a guiding principle, all of the physical treatments that have been shown to be effective in obsessivecompulsive disorder (OCD) (preferably in randomised, controlled trials) must have been tried in adequate dosage for an adequate period. In general terms, this will reflect the prescription of antidepressant drugs within, or above, the dose range recommended by the BNF for a period of 12-16 weeks. Most patients referred for assessment will have been exposed to many different treatment trials. The following represent those deemed essential before proceeding to neurosurgery.

(a) At least one course of treatment with the tricyclic antidepressant drug clomipramine for 16 weeks at a dosage in excess of $150 \mathrm{mg}$ per day. Except in exceptional circumstances, the dosage should be titrated upwards towards a target of $250 \mathrm{mg}$ per day (or above) depending on tolerability. Compliance ought to be determined by plasma level estimation where deemed necessary.

(b) At least two courses of treatment with different selective serotonin reuptake inhibitors (SSRIs) (fluoxetine, fluvoxamine, paroxetine, citalopram or sertraline) at the maximum tolerated dose for a period of 16 weeks. This may involve the prescription of these drugs at a dose in excess of the BNF maximum recommended dosage. Except in exceptional circumstances, all drugs from the SSRI class ought to be tried, sequentially, in full dosage (or maximum tolerated dosage) for an adequate period (the minimum target daily dose would be fluoxetine $60 \mathrm{mg}$, fluvoxamine $300 \mathrm{mg}$, sertraline $200 \mathrm{mg}$, citalopram $60 \mathrm{mg}$ and paroxetine $50 \mathrm{mg}$ ).

(c) At least one of the above plus lithium augmentation for a period of 12 weeks with a 12-h post-medication plasma lithium level of $0.4-0.8 \mathrm{mmol} / \mathrm{l}$.

(d) At least one of the above, plus neuroleptic augmentation for a period of 12 weeks (particularly where comorbid tic disorder or psychotic symptoms are present). Both typical and atypical agents may be tried (haloperidol and risperidone are recommended)

(e) At least one of the above plus augmentation with buspirone (10-60 mg per day), clonazepam (0.5$3.0 \mathrm{mg}$ per day) or nefazodone (200-600 mg per day) for a period of 12 weeks.

It is also anticipated that additional augmentation strategies may have been tried (e.g. L-tryptophan) Electroconvulsive therapy may be tried and becomes obligatory where depressive symptoms are prominent. The longer-term usefulness of intravenously administered clomipramine remains to be established.

Psychological treatment methods. At least one sustained trial (>26 weeks) of exposure and response prevention under the supervision of a $\mathrm{BABCP}$-accredited therapist (minimum therapist contact time $90 \mathrm{~min}$ per week). Whenever possible, we would expect a period (12 weeks) of in-patient behavioural therapy, conducted in a specialist unit. However, many sufferers are unwilling, for a variety of reasons, to consent to this. Cognitive therapy can also be an effective adjunct to exposure treatment if intrusive thoughts and ruminations are prominent. Again, trials of cognitive therapy ought to be conducted under the supervision of a BABCPaccredited therapist.

\section{REFERENCES}

Ballantine, H.T., Bouckoms, A. J., Thomas, E. K., et al (1987) Treatment of psychiatric illness by stereotactic cingulotomy. Biological Psychiatry, 22, 807-819.

Bridges, P. K. \& Goktepe, E. O. (1973) A review of patients with obsessional symptoms treated by psychosurgery. In Surgical Approaches in Psychiatry (eds L.V. Laitinen \& K. E. Livingstone), pp. 96-100. Baltimore, MD: University Park Press.

\section{_ , Bartlett, J. R., Hale, A. S., et al (1994)}

Psychosurgery: stereotactic subcaudate tractotomy. An indispensable treatment. British Journal of Psychiatry, 165 599-611.

Cosyns, P., Caemaert, J., Haaijman, W., et al (1994) Functional stereotaxic neurosurgery for psychiatric disorders: an experience in Belgium and the
Netherlands. Advances and Technical Standards in Neurosurgery, 21, 239-279.

CRAG Working Group on Mental IIIness (1996) Neurosurgery for Mental Disorder. A Report by a Good Practice Group of the CRAG Working Group on Mental Illness. Scottish Office J2318, 7/96. Edinburgh: HMSO.

Dougherty, D. D., Baer, L., Cosgrove, G. R., et al (2002) Prospective long-term follow-up of 44 patients who received cingulotomy for treatment-refractory obsessive-compulsive disorder. American Journal of Psychiatry, 159, 269-275.

Drevets, W. C., Price, J. L., Simpson, J. R., et al (1997) Subgenual prefrontal cortex abnormalities in mood disorders. Nature, 386, 824-827.

Goktepe, E. O., Young, L. B. \& Bridges, P. K. (1975) A further review of the results of stereotactic subcaudate tractotomy. British journal of Psychiatry, 126, 270-280.

Happe, F., Malhi, G. S. \& Checkley, S. (200I)

Acquired mind-blindness following frontal lobe surgery? A single case study of impaired 'theory of mind' in a patient treated with stereotactic anterior capsulotomy. Neuropsychologia, 39, 83-90.

Hay, P., Sachdev, P., Cummings, S., et al (1993) Treatment of obsessive compulsive disorder by psychosurgery. Acta Psychiatrica Scandinavica, 87 197-207.

Herner, T. (1961) Treatment of mental disorders with frontal stereotactic thermolesions. A follow-up of 116 cases. Acta Psychiatrica Scandinavica Supplementum, 37 158.

Hrobjartsson, A. \& Gotzsche, P. C. (200I) is the placebo powerless? An analysis of clinical trials comparing placebo with no treatment. New England Journal of Medicine, 344, 1594-1602.

Irle, E., Exner, C., Theilen, K., et al (1998) Obsessivecompulsive disorder and ventromedial frontal lesions: clinical and neuropsychological findings. American Journal of Psychiatry, I55, 255-263.

Jenike, M. A., Baer, L., Ballantine, H. T., et al (199I) Cingulotomy for refractory obsessive-compulsive disorder. A long-term follow-up of 33 patients. Archives of General Psychiatry, 48, 548-555.

Judd, L. L. (1997) The clinical course of unipolar major depressive disorders. Archives of General Psychiatry, 54, 989-991.

Kartsounis, L. D., Poynton, A., Bridges, P. K., et a (1991) Neuropsychological correlates of stereotactic subcaudate tractotomy. Brain, I14, 2657-2673.

Kelly, D. (1980) Anxiety and Emotions: Physiological Basis and Treatment. Springfield, IL: Charles Thomas.

Kiloh, L. G., Smith, J. S. \& Johnston, G. F. (1988) Psychosurgery (limbic surgery). In Physical Treatments in Psychiatry (eds L. G. Kiloh, J. S. Smith \& G. F. Johnston) pp. 277-333. Melbourne: Blackwell.

Knight, G. (1965) Stereotactic tractotomy in the surgical treatment of mental illness. Journal of Neurology, Neurosurgery and Psychiatry, 28, 304-310.

Lippitz, B. E., Mindus, P., Meyerson, B. A., et al (1999) Lesion topography and outcome after thermocapsulotomy or gamma knife capsulotomy for obsessive-compulsive disorder: relevance of the right hemisphere. Neurosurgery, 44, 452-458.

Malhi, G. \& Bartlett, J. R. (1998) A new lesion for the psychosurgical operation of stereotactic subcaudate tractotomy (SST). British Journal of Neurosurgery, 12 , 335-339.

Marks, I. M., Birley, J. L. \& Gelder, M. G. (1966) Modified leucotomy in severe agoraphobia: a controlled serial inquiry. British Journal of Psychiatry, II2, 757-769. 
Mataix-Cols, D., Rauch, S. L., Baer, L., et al (2002) Symptom stability in adult obsessive-compulsive disorder: data from a naturalistic two-year follow-up study. American Journal of Psychiatry, 159, 263-268.

Matthews, K. \& Eljamel, M. S. (200I) Neurosurgery for Mental Disorder in Dundee. http: / /www.show.scot. nhs.uk/mhwbsg/Documents/NMDReport.pdf.

Millan Committee (200I) New Directions: Review of the Mental Health (Scotland) Act 1984. Scottish Executive Publications SE/2001/56. Edinburgh: Stationery Office.

Mindus, P. \& Nyman, H. (1991) Normalization of personality characteristics in patients with incapacitating anxiety disorders after capsulotomy. Acta Psychiatrica Scandinavica, 83, 283-291.

Moniz, E. (1936) Essai, d'un traitement chirurgical de certaines psychoses. Bulletin de l'Académie de Médecin, II5, 385-392.

Nuttin, B., Gybels, J., Cosyns, P., et al (1999) Electrical stimulation in anterior limbs of internal capsules in patients with obsessive-compulsive disorder. Lancet, 354, 1526.

Ochsner, K. N., Kosslyn, S. M., Cosgrove, G. R., et al (200I) Deficits in visual cognition and attention following bilateral anterior cingulotomy. Neuropsychologia, 39 219-230

Ongur, D., Drevets, W. C. \& Price, J. L. (1998) Glial reduction in the subgenual prefrontal cortex in mood disorders. Proceedings of the National Academy of Sciences of the USA, 95, 13290-13295.

Poynton, A. M., Kartsounis, L. D. \& Bridges, P. K. (1995) A prospective clinical study of stereotactic subcaudate tractotomy. Psychological Medicine, 25, 763-770.

Rajkowska, G., Miguel-Hidalgo, J. J., Wei, J., et al (1999) Morphometric evidence for neuronal and glial prefrontal cell pathology in major depression. Biological Psychiatry, 45, 1085-1098.

Royal College of Psychiatrists (2000) Neurosurgery for Mental Disorder. Report from the Neurosurgery Working Group of the Royal College of Psychiatrists (Council Report CR89). London: Royal College of Psychiatrists.

Rush, A. J., George, M. S., Sackeim, H. A., et al (2000) Vagus nerve stimulation (VNS) for treatment resistant depression: a multicenter study. Biological Psychiatry, 47, 276-286.

Sackeim, H. A., Rush, A. J., George, M. S., et a (200I) Vagus nerve stimulation (VNS) for treatmentresistant depression: efficacy, side effects and predictors of outcome. Neuropsychopharmacology, 25, 713-728.

Sheline, Y. I., Wang, P. O., Gado, M. H., et al (1996) Hippocampal atrophy in recurrent major depression. Proceedings of the National Academy of Sciences of the USA, 63, 3908-3913.

\section{CLINICAL IMPLICATIONS}

- Modern neurosurgery for mental disorder bears little relation to the early procedures and clinical practice that brought discredit upon psychiatry.

- Ablative neurosurgery remains available as a treatment option for chronic, intractable depression and obsessive-compulsive disorder.

- Despite developing empirically, modern ablative neurosurgery targets neural structures that have been shown to exhibit functional and structural abnormalities and cannot be considered to represent 'healthy' tissue.

\section{LIMITATIONS}

- The evidence base for ablative neurosurgery for mental disorder remains unsatisfactory.

- A plausible mechanism of action for such neurosurgery has yet to be identified.

- The importance of defining and describing key features of postoperative management has been neglected.

KEITH MATTHEWS, MD, PhD, MRCPsych, MUFTAH S. ELJAMEL, MD, FRCSI, FRCS(NS), FABI, Ninewells Hospital and Medical School, Dundee, Scotland, UK

Correspondence: Professor Keith Matthews, Department of Psychiatry, Ninewells Hospital, Dundee DDI 9SY,UK. Tel: 01382 632121; fax: 01382 633923; e-mail: k.matthews@dundee.ac.uk

(First received 8 November 200I, final revision 23 October 2002, accepted 12 November 2002)

Skoog, G. \& Skoog, l. (1999) A forty-year follow-up of patients with obsessive compulsive disorder. Archives of General Psychiatry, 56, 121-127.

Spangler, W., Cosgrove, R., Ballantine, H.T., et al (1996) Magnetic resonance image-guided stereotactic cingulotomy for intractable psychiatric disease. Neurosurgery, 38, 107|-1078.

Szeszko, P. R., Robinson, D., Alvir, J., et al (1999) Orbital frontal and amygdala volume reductions in obsessive compulsive disorder. Archives of General Psychiatry, 56, 913-919.

Tan, E., Marks, I. M. \& Marset, P. (197I) Bimedia leucotomy in obsessive-compulsive neurosis: a controlled serial inquiry. British Journal of Psychiatry, II8, 155-164.

Vilkki, J. (1977) Late psychological and clinical effects of subrostral cingulotomy and anterior mesoloviotomy in psychiatric illness. In Neurosurgical Treatment in Psychiatry, Pain and Epilepsy (eds W. H. Sweet, S. Obrador \& J. Martin-Rodriguez), pp. 253-259. Baltimore, MD: University Park Press.

Waziri, R. (1990) Psychosurgery for anxiety and obsessive-compulsive disorders. In Handbook of Anxiety (vol. 4) (eds R. Noyes, M. Roth \& G. Burrows). Amsterdam: Elsevier. 